\title{
Contractility-induced self-organization of smooth muscle cells: from multilayer cell sheets to dynamic three-dimensional clusters
}

\author{
Xiuyu Wang ${ }^{1}$, David Gonzalez-Rodriguez ${ }^{2}$, Thomas Vourc' ${ }^{3+}$, \\ Pascal Silberzan ${ }^{3}$, Abdul I. Barakat ${ }^{*}$
}

${ }^{1}$ Hydrodynamics Laboratory - CNRS UMR 7646, Ecole Polytechnique, Institut Polytechnique de Paris, Palaiseau, France

${ }^{2}$ Université de Lorraine, LCP-A2MC, Metz, France

${ }^{3}$ Laboratoire PhysicoChimie Curie, Institut Curie, PSL Research University, Paris, France

${ }^{+}$Current Address: Université Clermont Auvergne, SIGMA Clermont, Institut Pascal, BP 10448, F-63000 Clermont-Ferrand, France

* Correspondence: Prof. Abdul I. Barakat - abdul.barakat@polytechnique.edu 


\begin{abstract}
Smooth muscle cells (SMCs) are mural cells that play a vital contractile function in many tissues. Abnormalities in SMC organization are associated with many diseases including atherosclerosis, asthma, and uterine fibroids. Various studies have reported that SMCs cultured on flat surfaces can spontaneously form three-dimensional clusters whose organization resembles that encountered in some of these pathological settings. Remarkably, how these structures form remains unknown. Here we combine in vitro experiments and physical modeling to show that three-dimensional clusters initiate when cellular contractile forces induce a hole in a flat SMC sheet, a process that can be modeled as the brittle fracture of a viscoelastic material. The subsequent evolution of the nascent cluster can be modeled as an active dewetting process with cluster shape evolution driven by a balance between cluster surface tension, arising from both cell contractility and adhesion, and cluster viscous dissipation. The description of the physical mechanisms governing the spontaneous emergence of these intriguing three-dimensional clusters offers insight into SMC-related disorders.
\end{abstract}

Key words: smooth muscle cells, cluster formation, collective behavior, dewetting, brittle fracture, cellular organization 


\section{Introduction}

Smooth muscle cells (SMCs) are contractile cells that populate the walls of many hollow tissues and organs including blood vessels, pulmonary airways, and the gastrointestinal and urogenital systems $^{1,2}$. In vivo, SMCs regulate tissue tone and synthesize a host of extracellular matrix proteins. Aberrant SMC proliferation and organization are the hallmarks of many chronic pathologies including atherosclerosis ${ }^{3}$, hypertension ${ }^{4}$, asthma ${ }^{5}$, uterine fibroids ${ }^{6}$ (UFs), and obstructive gastrointestinal diseases ${ }^{7}$. In normal tissue, SMCs are typically organized in a multilayered structure with highly aligned cells whose architecture is tailored for optimal contractile function. Abnormalities in this organization are associated with a number of disorders. A prominent example is the case of UFs, common benign smooth muscle tumors that afflict up to $60-80 \%$ of all women ${ }^{8}$, where SMCs organize into three-dimensional spheroid-like structures. The factors driving the changes in SMC organization during UF formation remain unclear.

A number of studies over the past few decades have reported that SMCs cultured on flat surfaces can under certain conditions spontaneously form three-dimensional structures that have alternately been referred to as hills, mounds, or hill-and-valley (HV) patterns ${ }^{9-11}$. These structures have been reported in SMCs from different species including human ${ }^{12}$, pig $^{13}$, rat $^{14-16}$, and cow ${ }^{17}$. In some cases, the HV patterns transition to spherical "ball-like" clusters ${ }^{18}$ that can either remain adherent to the substrate from which they emerged or become suspended in the culture medium. Interestingly, ball-like structures that originate from SMCs derived from human UFs revert back to HV patterns upon treatment with gonadotrophin releasing hormone $(\mathrm{GnRH})$ in culture ${ }^{19}$, suggesting that these three-dimensional structures may constitute a useful in vitro model for fibroids. Remarkably, despite the repeated and widespread observations, the physical mechanisms governing the spontaneous emergence of these intriguing threedimensional SMC clusters and the dynamics of their evolution remain unexplained.

Here we use a combination of in vitro experiments and physical modeling to elucidate the mechanisms governing the spontaneous emergence and dynamics of three-dimensional SMC clusters from flat two-dimensional SMC layers. We show that the clusters initiate when SMC contractile forces overcome adhesion forces to the substrate, which only occurs once a critical SMC density has been attained. We also show that cluster formation occurs through a precise sequence of physical events whose dynamics we characterize both experimentally and using models that rely upon the well-established analogy between the spreading of cellular aggregates and the dewetting phenomena of liquid droplets ${ }^{20,21}$.

\section{Results}

Culturing SMCs on a fibronectin-coated flat glass surface leads to the spontaneous emergence of three-dimensional clusters once a sufficiently high cell density is attained (Supplementary Movie 1). The cluster diameter varies over a wide range of $\sim 100 \mu \mathrm{m}$ to $\sim 3 \mathrm{~mm}$. As shown in Fig. 1a and schematized in Fig. 1c, SMCs within clusters exhibit two distinct patterns of organization. Along the peripheral region of the cluster, particularly in the bottom portion, cells are oriented radially, have elongated nuclei, and express abundant F-actin, consistent with radial force generation associated with the anchoring to the surrounding SMC layer. On the other hand, SMCs in the inner region of the cluster assume an architecture that resembles a bird nest with 
nuclei oriented circumferentially and strikingly low levels of F-actin expression, suggesting minimal force exertion. Interestingly, the centermost portion of the cluster often contains a zone of lower cell density (Fig. 1a, b) that spans a portion of the height of the cluster. Live/dead staining revealed that the vast majority of the cells were viable (Supplementary Fig.1).
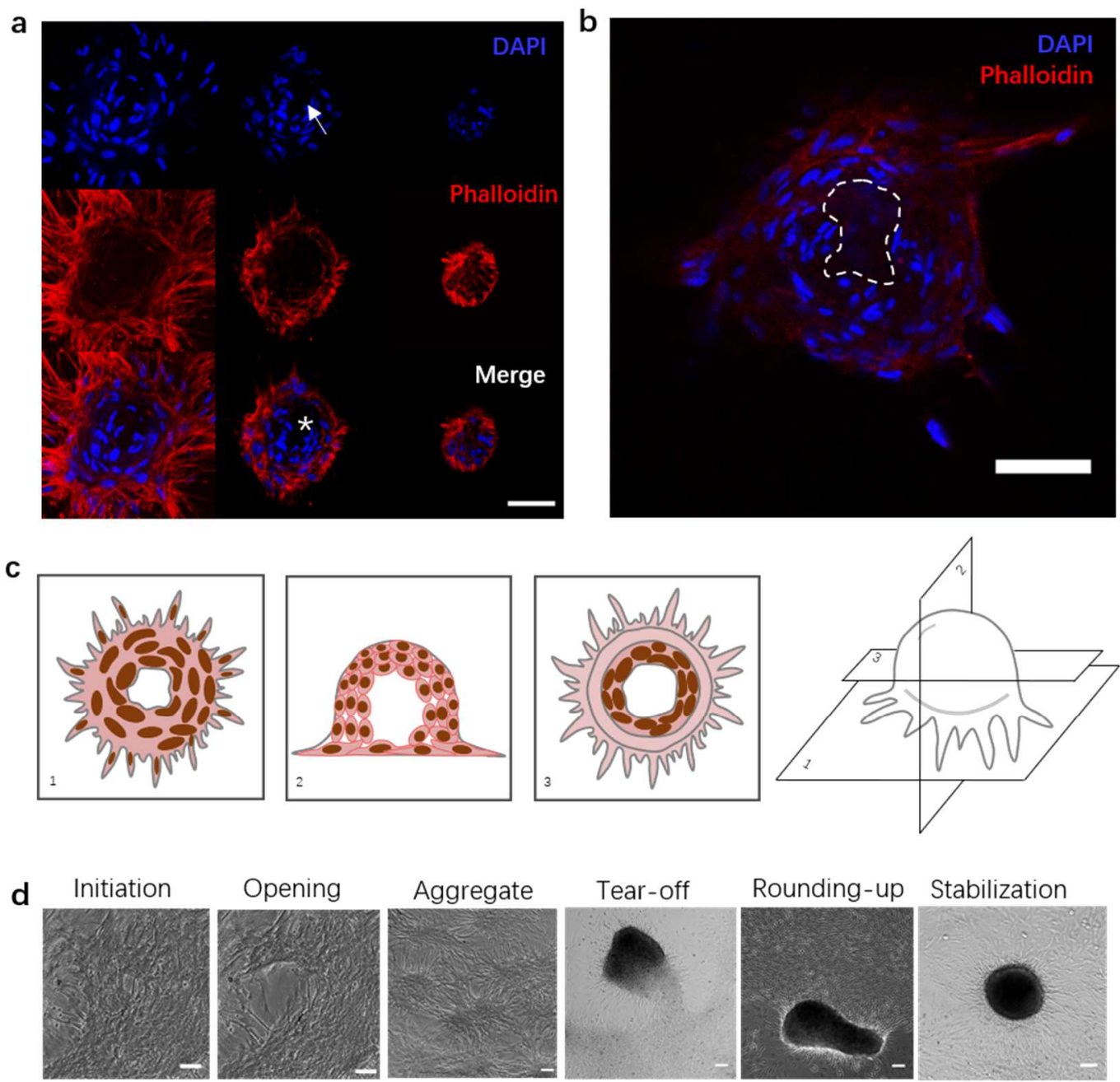

Figure 1. Cellular organization in SMC clusters and sequence of steps leading to cluster formation. a: Confocal images of cellular organization within a cluster. From left to right: zstack from bottom to top of a cluster for the nucleus (DAPI channel; top row), F-actin (phalloidin channel; middle row), and both channels combined (bottom row). Note the lower cell density zone in the center of the cluster (white arrow on DAPI image; white * on merge image). Scale bar: $50 \mu \mathrm{m}$. b: A confocal optical section through the midplane of a cluster to more clearly illustrate the lower cell density zone in the center (dashed contour). Scale bar: 50 $\mu \mathrm{m}$. c: Schematic of cellular organization within SMC clusters. d: Phase contrast images of the sequence of five distinct events leading to cluster formation. From left to right: initial condition, crack development, aggregate formation, aggregate tear-off, cluster rounding-up from oblong to circular cross-section, and cluster stabilization. Scale bars: $100 \mu \mathrm{m}$.

Time-lapse imaging revealed that cluster formation occurs through the following sequence of specific physical events (Fig. 1d): 1) contractility-induced formation of a breach or hole in the flat SMC layer (Supplementary Movie 2), presumably due to an imbalance in the exerted 
contractile forces as a result of a locally elevated SMC concentration; 2) cellular retraction after hole formation, leading to a bumpy SMC "aggregate" that nevertheless remains fully attached to the substrate over its entire contour (Supplementary Movie 3); 3) additional contraction of the aggregate until a portion of it tears off from the substrate, giving rise to a well-defined threedimensional cluster (Supplementary Movie 4); 4) rounding-up of the typically oblong cluster into a hemispherical shape (Supplementary Movie 5); 5) cluster stabilization in its final hemispherical shape until the end of the observation (Supplementary Movie 6). Two clusters that come in contact are sometimes observed to fuse into a single cluster (Supplementary Movie 7). The physics of each of the five steps outlined above are described next.

Hole development in the SMC layer and aggregate formation. We investigated cluster formation at three different SMC seeding densities: low, medium and high corresponding respectively to $40,000,80,000$, and $160,000 \mathrm{cells} / \mathrm{cm}^{2}$ (see Methods). For the high-density group, cluster formation initiates within $24 \mathrm{~h}$ of seeding with the spontaneous development of holes in the SMC layer (Fig. 2a). In the early stages, the hole may still be partially covered with cells under high strain due to contractile forces from surrounding cells (Fig. 2a).

We quantified the velocity field in the cell sheet surrounding the hole using particle image velocimetry (PIV) (Fig. 2b, c). The hole widens as cells pull in opposite directions with a progressively increasing velocity. When the cell velocity on either side of the hole attains a peak (typically $\sim 150 \mu \mathrm{m} / \mathrm{h}$ ), the cells extending over the hole tear off, which has the effect of releasing the tension and stabilizing the hole width. This is accompanied with an overall reduction in cell velocity below $\sim 20 \mu \mathrm{m} / \mathrm{h}$.

We also characterized the evolution of the size and shape of the hole (Fig. 2d-f). This evolution can be modeled as the brittle fracture of a viscoelastic material where the dynamics are dominated by elasticity, similar to the work of Tabuteau et al. ${ }^{22}$ The profile of the hole can be described by a parabola of equation $y=A \sqrt{x}$, where the symmetry axis $y$ of the parabola is aligned with the direction of hole propagation and the constant $A \sim \sqrt{\frac{G}{E}}$ depends on the adhesion energy $G \sim 10^{-2} \mathrm{~J} / \mathrm{m}^{2} 23$ and the SMC elastic modulus $E \sim 10^{4} \mathrm{~Pa}^{24}$. Modeling the cell sheet as an elastic sheet, the opening dynamics of the hole width $a$ are governed by a balance between cell elastic recoil and friction with the substrate, which leads to equation (1):

$$
a=a_{\max }[1-\exp (-C t)]
$$

where $C \sim \frac{E}{k R_{c}}$ is a constant, $k$ is the cell-substrate friction, and $R_{\mathrm{c}}$ is the cell size. By fitting the experimental data (Fig. 2e), we obtain experimentally $a_{\max } \sim 200 \mu \mathrm{m} \sim R_{c}$ and $C \sim 2 \cdot 10^{-3} \mathrm{~s}^{-1}$, which enables us to estimate a cell-substrate friction coefficient of $k \approx 2 \cdot 10^{10} \mathrm{~Pa}_{\mathrm{s} . \mathrm{m}^{-1}}$, comparable to albeit slightly larger than a friction coefficient value reported previously ${ }^{25,26}$. Along its length, the hole opens in successive phases, corresponding approximately to one cell length ${ }^{27}$ (Fig.2f). According to our theoretical interpretation, the time to advance one phase should then scale as the stretching time $1 / \mathrm{C} \sim 500 \mathrm{~s}$, which is consistent with the observations. This hole development phenomenon is strongly reminiscent of the dewetting of a cell monolayer described by Douezan et al. ${ }^{28}$, although here the dominant driving force is cell elastic 
recoil, rather than intercellular adhesion.

An established hole represents a valley in the commonly described HV SMC pattern. Once the hole expands, it ultimately forms a ring, leading to a topological transition with the formation of an "aggregate", a multi-layer hill-like structure with a dense central portion and radially outward-pointing cells at the periphery (Supplementary Movie 3 and Supplementary Fig. 2a). These aggregates are highly dynamic as a result of gradients in cell pulling forces. PIV analysis revealed considerably higher cellular velocities in the monolayer $(\sim 30 \mu \mathrm{m} / \mathrm{h})$ opposite to the direction of aggregate movement (Supplementary Fig. 2b, c).
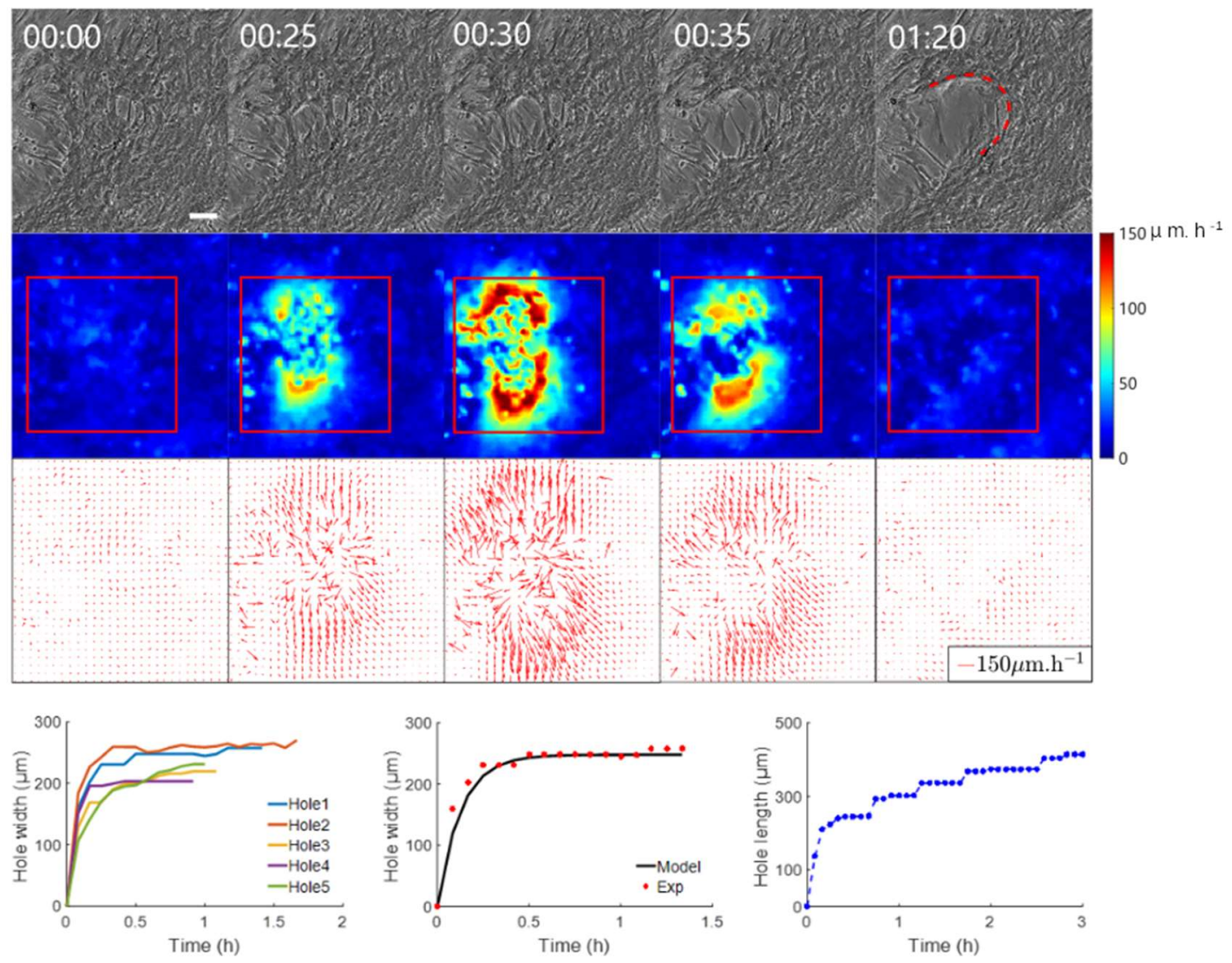

Figure 2. Dynamics of hole development. a: Phase contrast images of SMCs at different times pre and post hole development. White arrow denotes the position of hole initiation. Fit of the experimental shape of the hole edge by a parabola (red curve) whose equation is indicated in the text. Times are in hours and minutes. Scale bar: $100 \mu \mathrm{m}$. b: Heat map of SMC tissue speed corresponding to the velocity field shown in panel c. c: Velocity vector field in the SMC tissue showing the larger velocities in the hole vicinity. $\mathbf{d}$ : Time evolution of hole width for five different holes. e: Comparison between the mathematical model of hole width evolution (solid black curve) and experimental data (red circles). f: Length development for one representative hole. Note the successive phases of hole opening.

Cluster rounding and stabilization. Driven by cell contraction, aggregates subsequently tear off from the substrate and form independent clusters (Supplementary Fig. 3). These clusters are mobile on the substrate as they progressively round up (Fig. 3a). We analyzed the evolution of 
cluster size and shape during this rounding-up process as well as cluster motion (Supplementary Fig. 4 and Supplementary Note 1) by imaging the cluster every 20 min (Supplementary Note 2). Cluster morphometric data were obtained through automatic detection of the bright contour in the images (Fig. 3a). Over $24 \mathrm{~h}$, the cluster projected area decreases by about $40 \%$ and its circularity (defined as $4 \pi A / P^{2}$, where $\mathrm{A}$ is the cluster area and $\mathrm{P}$ is its perimeter), increases from 0.65 to almost 1 (Fig. 3b, c), thus indicating that the cluster becomes more compact and round over time. Similar to previous models ${ }^{29}$, we interpret the dynamics of rounding up as a phenomenon driven by cluster surface tension, arising from both cell contractility and adhesion, and resisted by cluster viscous dissipation. Mathematically,

$$
\gamma \dot{S}=-\eta \int_{V} \vec{\nabla} \vec{u}: \vec{\nabla} \vec{u} \mathrm{~d} V
$$

where $\gamma$ is the surface tension coefficient, $\eta$ the cluster viscosity, $S$ and $V$ the cluster surface area and volume, and $\vec{u}$ is the velocity field within the cluster. We assume the cluster shape to be a prolate spheroid of major axis $a$ and minor axis $c$. By considering the spheroid to be sufficiently close to a sphere, we can obtain an expression for the velocity field and obtain a closed-form approximate solution to equation (2) (Supplementary Note 3):

$$
\operatorname{In}\left(\frac{\epsilon}{\epsilon_{0}}\right)=-\frac{15}{56} * \frac{\gamma}{\eta} * \frac{t}{r}
$$

where we have defined $\epsilon=\frac{(c-a)}{c+a}$ and $r=\frac{2 a+c}{3}$.

A fit of Eq. (3) to the experimental data is shown in Fig. 3d, yielding $\frac{\gamma}{\eta} \approx 10^{-8} \mathrm{~m} / \mathrm{s}$, comparable to that reported for the rounding up of cellular aggregates of other cell types ${ }^{28}$. Once clusters round up, they stabilize in size and shape (Supplementary Fig. 5, Supplementary Note 4) unless they fuse with other clusters.

a

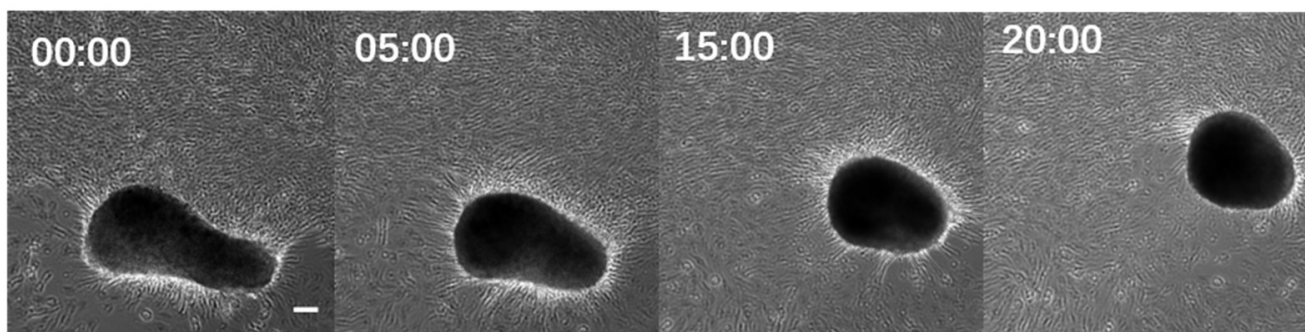

b

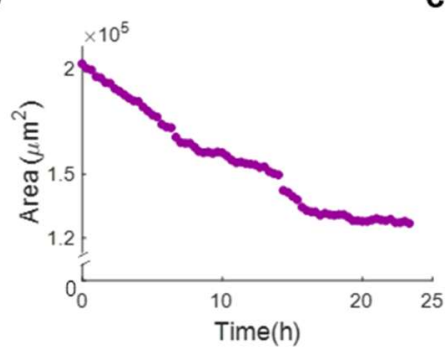

C

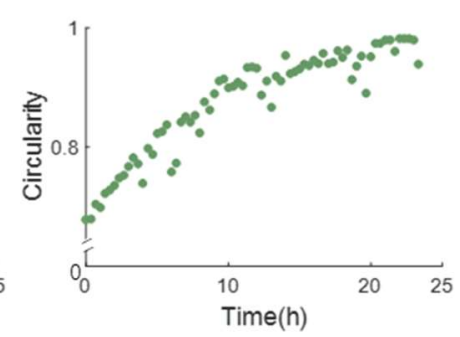

d

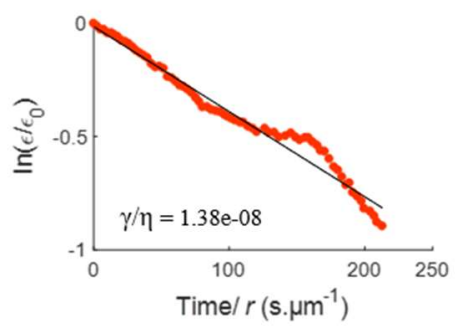

Figure 3. Cluster rounding dynamics. a: Phase contrast images of a SMC cluster at different times showing progressive rounding. Times are in hours. Scale bar: $100 \mu \mathrm{m}$. b: Time evolution of cluster projected area. c: Time evolution of cluster circularity. d: Normalized cluster/spheroid anisotropy, allowing determination of the value of the ratio of surface tension 
to viscosity $(\gamma / \eta)$. Equation is indicated in the text.

Cluster fusion. The interaction of two clusters that are sufficiently close to one another often leads to fusion (Fig. 4a). The fused cluster subsequently goes through the "rounding-up" and "stabilization" steps described above for single clusters.

To analyze the fusion process, we quantify the evolution of the "neck" radius $\rho$, i.e., the radius of the contact region between two adjacent clusters as seen in projection on the experimental images. The observed neck evolution is reasonably fitted by equation (4) of the sintering theory proposed by Douezan and Brochard ${ }^{29}$ :

$$
\frac{\rho^{2}}{R_{0}}=\frac{\gamma}{\eta} * t
$$

Here, $R_{0}$ is the cluster radius, $R_{0}=\operatorname{sqrt}\left(\frac{A_{1}+A_{2}}{2 * \pi}\right)$, with $\mathrm{A}_{1}$ and $\mathrm{A}_{2}$ the initial cluster areas, $\gamma / \eta$ is the ratio of surface tension to viscosity, and $t$ is the elapsed time since the beginning of the fusion process (Fig. $4 \mathrm{~b}, \mathrm{c}$ ). By fitting the experimental observations of aggregate fusion, we obtain a value of $\gamma / \eta$ of the order of $1-3 \times 10^{-9} \mathrm{~m} / \mathrm{s}$. Taking into account possible scaling factors that arise from our simplified geometrical treatment of the problem, this value of $\gamma / \eta$ appears consistent with that obtained for rounding-up. Our findings thus suggest that both phenomena, "rounding-up" and "fusion", are governed by the same physics of an effective surface tension driving the morphological change, whose speed of evolution is limited by viscous dissipation.

a

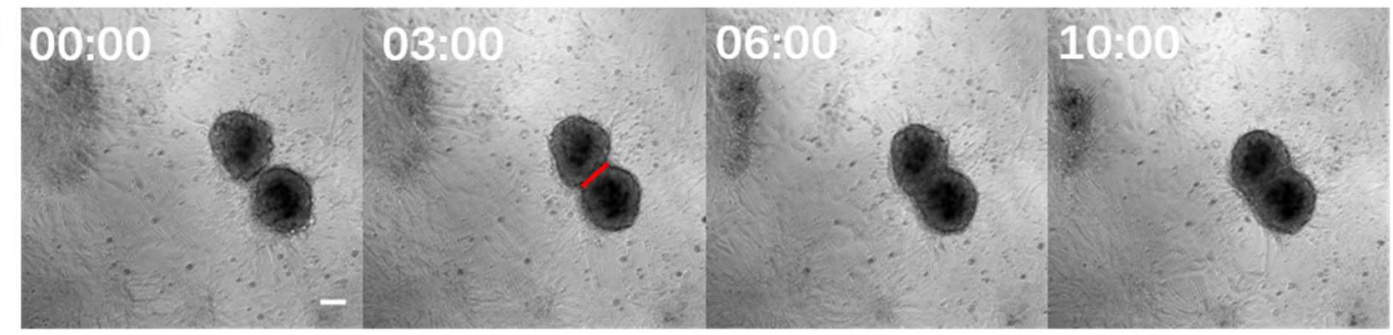

b

C
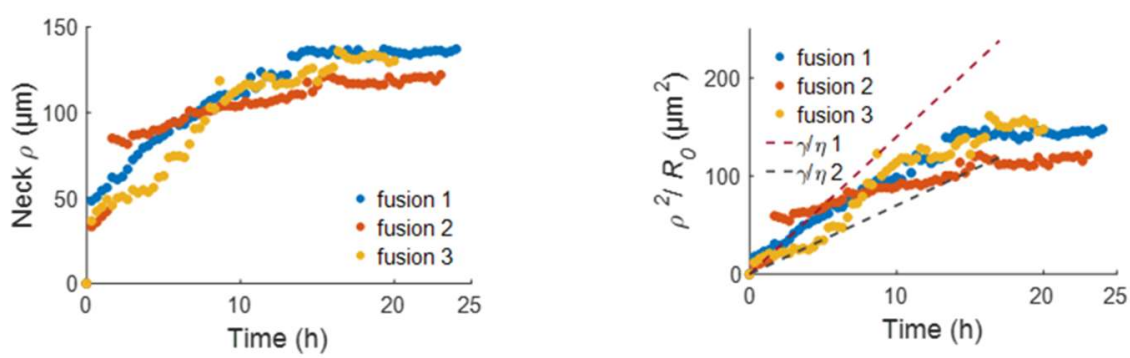

Figure 4. Cluster fusion. a: Phase contrast images of two fusing SMC clusters at different times. Times are in hours. Red line: $2 \rho$, where $\rho$ is the "neck" radius. Scale bar: $100 \mu \mathrm{m}$. b: Time evolution of "neck" radius $\rho$. c: Time evolution of the normalized neck radius. $\gamma / \eta 1=$ $3.5 \times 10^{-9} \mathrm{~m} / \mathrm{s}, \gamma / \eta 2=1.2 \times 10^{-9} \mathrm{~m} / \mathrm{s}$. Equation is indicated in the text.

\section{Prerequisite conditions for SMC cluster formation.}

The occurrence of SMC cluster formation as detailed above requires two prerequisite conditions: 
a sufficiently high cell density and sufficient levels of SMC contractility. The role of each of these two conditions is characterized next.

Role of cell density. SMC density determines both the dynamics of cluster formation and the pre-cluster organization of SMC sheets necessary for initiating a cluster.

(i) Dynamics of cluster formation: We investigated the dynamics of cluster formation for the three different cell seeding densities tested $\left(40,000,80,000\right.$, and 160,000 cells $\left./ \mathrm{cm}^{2}\right)$ (Supplementary Fig. 6). We divide the clusters into two types (Supplementary Fig. 7): "developed clusters", which have a clear and complete contour, and "developing clusters", which have a partially developed contour. Not surprisingly, cluster formation occurs most rapidly at the highest cell seeding density for both types of clusters (Fig. 5a, b and Supplementary Fig. 6b). Furthermore, cluster formation at the lower seeding densities occurs only after a delay, suggesting that a critical cell density needs to be attained via cell proliferation for cluster formation to initiate. For both types of clusters, the number of clusters increases for several days until it reaches a maximum and then decreases before ultimately stabilizing at a plateau. For developing clusters, the increase is due to cluster formation because of cell proliferation, whereas the decrease corresponds to developing clusters maturing into developed clusters. For developed clusters, the increase is driven by the maturing developing clusters, while the decrease is due to cluster fusion once cluster density becomes sufficiently high for neighboring clusters to interact. The time evolution of the total number of clusters (i.e. developing and developed) exhibits similar overall behavior (Fig. 5c) once a shift of 3 days has been introduced for the low and medium seeding densities, so that $t=0$ for all experiments is the time when cluster formation starts. With this shift, all experimental curves are approximately similar. This master curve first rapidly increases to reach a maximum, and then decreases to finally stabilize at a plateau.

The master curve in Fig. 5c can be described by the following population dynamics differential equation (5):

$$
\frac{\mathrm{d} N}{\mathrm{~d} t}=p_{1}-\alpha(t) p_{2} N,
$$

where $N$ is the total number of clusters at time $t, p_{1}$ is the rate of cluster formation, and $p_{2}$ the rate of (relative) cluster number reduction, mainly arising from cluster fusion. The timedependent parameter $\alpha(t)$ is introduced to account for a reduced rate of cluster number at short time, due to the time required for clusters to meet each other and fuse. We assume a linear increase of the dissociation rate over time, $\alpha(t)=\min \left(t / t_{\mathrm{s}}, 1\right)$, with $t_{\mathrm{s}}$ being a certain stabilization time. The solution to this differential equation agrees closely with the experimentally observed shape. Figure $5 \mathrm{c}$ shows a comparison between the computational fit and the experimental data with fitted model parameter values of $p_{1}=50$ clusters/day, $p_{2}=1.5$ day $^{-1}$ and $t_{\mathrm{s}}=8$ days. 

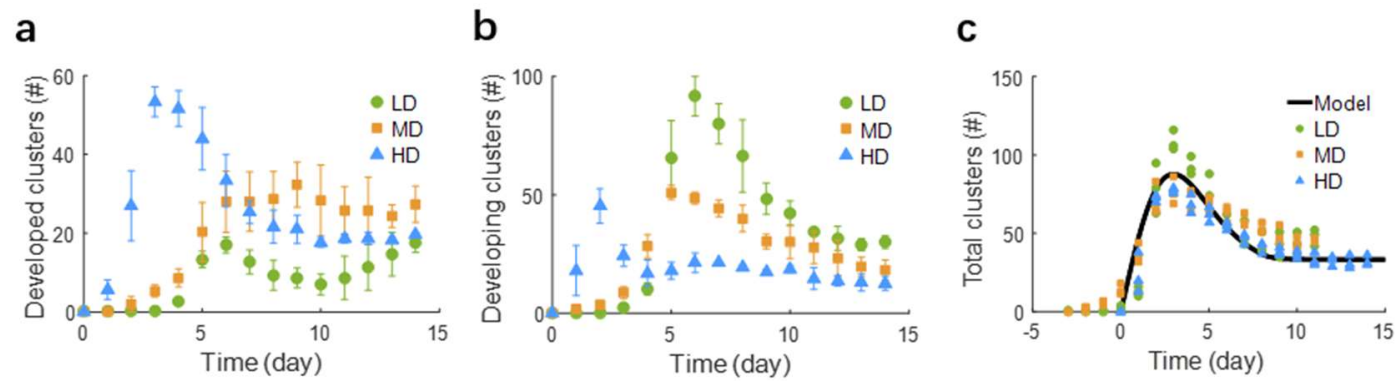

Figure 5. Dynamics of cluster evolution. a: Time evolution of the number of developed clusters for the different cell seeding densities. The maximum number of developed clusters is reached at days 14, 9 and 4 for the low (LD), medium (MD), and high (HD) seeding densities, respectively. Error bars are SEM of three independent experiments. b: Time evolution of the number of developing clusters for the different cell seeding densities. The maximum number of developing clusters is reached at days 6, 5 and 2 for the low (LD), medium (MD), and high (HD) seeding densities, respectively. Error bars are SEM of three independent experiments. c: Time evolution of total number of clusters (sum of developed and developing clusters) and physical model fit. Three experiments for each density are shown.

(ii) Pre-cluster SMC organization: It would be instructive to characterize the organization of our SMC layers prior to cluster formation for the different cell densities. To this end, we quantify the extent of actin stress fiber alignment by computing the scalar order parameter $Q$ for non-polar particles ${ }^{30}$ (Supplementary Fig. 8):

$$
Q=\sqrt{\left.<\cos 2 \theta\rangle^{2}+<\sin 2 \theta\right\rangle^{2}}
$$

where $\theta$ is the angle between the stress fiber direction and a reference direction (the horizontal direction in this case). Q ranges from 0 for a completely disordered case to 1 for a perfect nematic order where all stress fibers are aligned in the same direction. At low cell density, SMCs exhibit a wide range of orientations (Fig. 6a) as indicated by the small value of $\mathrm{Q}=0.12$. At medium density, SMCs coordinate with local alignment of stress fibers, leading to considerably increased order $(\mathrm{Q}=0.69)$. At high density, SMCs rapidly form multiple layers, typically three (Fig. 6b), prior to cluster formation, and actin stress fibers within each layer are highly aligned (Fig. 6b, c), with an order parameter approximately equal to unity $(\mathrm{Q}=0.95)$. These findings are consistent with previous studies ${ }^{30-32}$. 


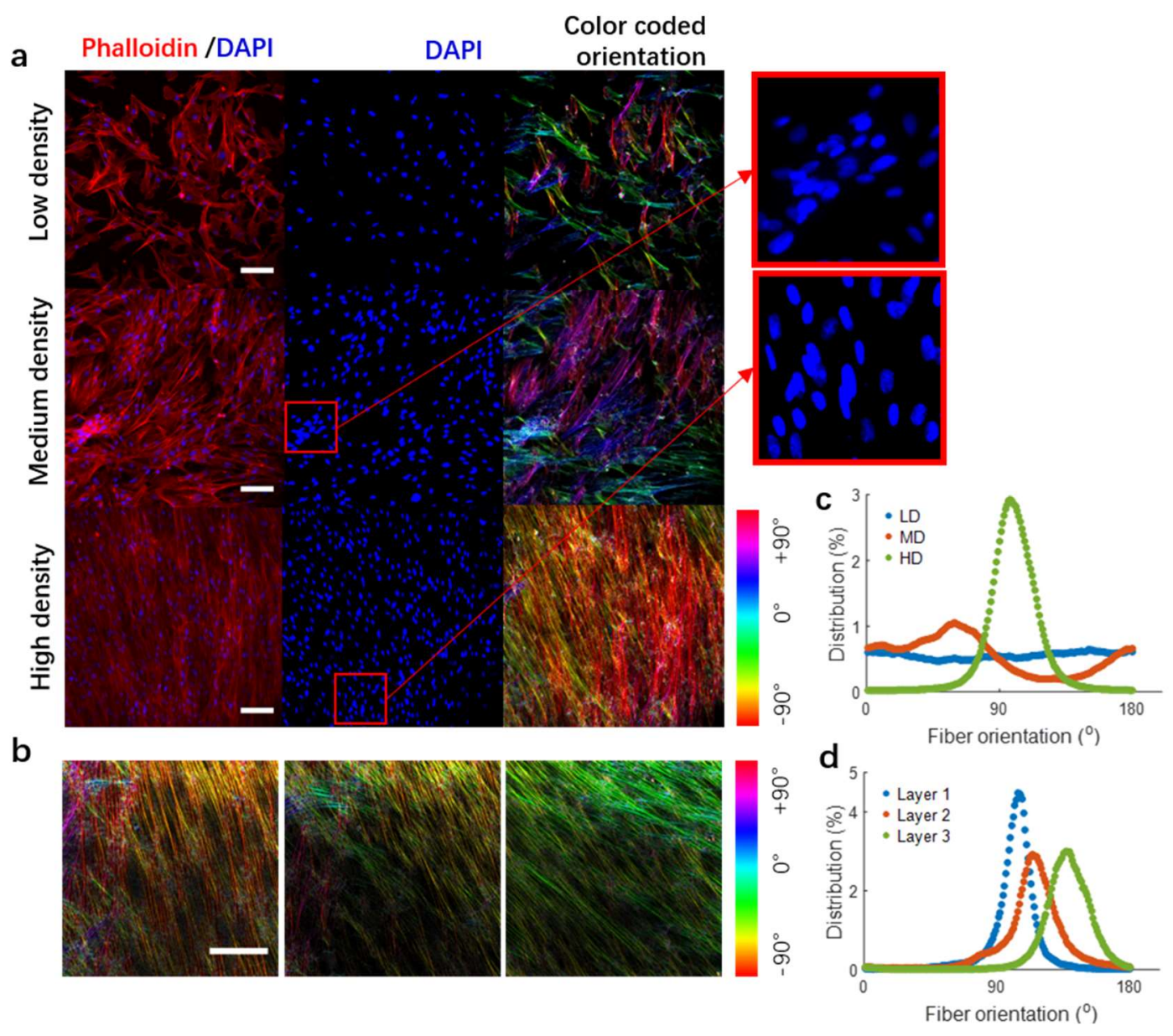

Figure 6. Pre-cluster SMC actin stress fiber organization. a: Immunostaining images of samples with different seeding density $24 \mathrm{~h}$ post-seeding. DAPI (blue) shows SMC nuclei, while phalloidin (red) shows F-actin. Nuclear overlap in some regions (see insets) demonstrates that cells begin to form local multi-layer zones at the medium density. Scale bars: $100 \mu \mathrm{m}$. b: Maps of F-actin orientation of one three-layer sample at high cell seeding density obtained by confocal microscopy. Left: bottom layer; middle: second layer; right: top layer. Scale bar: 50 $\mu \mathrm{m}$. c: F-actin orientation histograms for the low (LD), medium (MD), and high (HD) seeding density samples, showing progressively increasing orientation. d: F-actin orientation distributions for the three-layer sample in panel $b$.

Role of contractility. Our results indicate that SMC cluster formation only initiates once a critical cell density is attained and the cells have formed several layers. However, cell density per se is not sufficient to initiate cluster formation. The additional required component is a sufficiently elevated cell contractility in order to overcome cell-substrate adhesion as depicted schematically in Fig. S9a. Such force disequilibrium would lead to the tearing-off of the SMC cell sheet from the substrate. In support of this model, SMC contractility inhibition using blebbistatin $(5 \mu \mathrm{M})$ significantly slows down cell detachment from the substrate and delays the formation of clusters (Supplementary Fig. 9b), confirming the important role that SMC contractility plays in cluster formation. A higher dose of blebbistatin $(10 \mu \mathrm{M})$ further reduces the area of cellular detachment (more than 20 times smaller than the untreated controls). 
As an additional test, we investigated the dynamics of SMC cluster formation when the cells were cultured on very soft gels (Young's modulus of $\sim 2 \mathrm{kPa}$ ) on which their ability to generate large contractile forces is greatly diminished. The results demonstrated that when plated at the same seeding density, cluster formation on the soft gel required 3 days longer than on plastic (Young's modulus $\sim 2.7 \mathrm{GPa}$ ). Applying the model described in the "Rounding-up" section for the dynamics of cluster formation yields a value of $\gamma / \eta$ that is 3 to 4 times smaller for the soft gel than for plastic (Fig. S9c), consistent with a slower rate of cluster formation. These findings are consistent with the central role for contractility as the driving force for SMC cluster formation.

\section{Discussion}

The spontaneous formation of three-dimensional SMC clusters, referred to elsewhere as HV patterns and ball-like structures, from flat cellular layers has been described qualitatively in many studies over the past few decades; however, the physical processes giving rise to these clusters have not been described. The present study provides the first detailed quantitative characterization and physical modeling of the events leading to the formation of SMC clusters.

Our experiments indicate that cluster formation occurs through a sequence of physical events whose dynamics and underlying mechanisms we characterize by conceptualizing SMC clusters and their precursor structures (referred to as aggregates) as active liquid droplets. This physical formulation allows us to explain the observed dynamics of: (i) hole development in the flat SMC layer, analogous to brittle viscoelastic fracture followed by dewetting, (ii) cluster shape evolution, analogous to droplet rounding-up, and (iii) cluster fusion, analogous to droplet coalescence. The model's predictions are in agreement with observations and provide estimates of the material properties of SMC clusters. At a population scale, we describe the evolution of the number of clusters by a population dynamics model that accounts for cluster formation and cluster fusion.

Cells in SMC clusters are oriented radially along the periphery of the cluster and circumferentially in the interior region with a near "cell-free" zone in the center. This organization also shares some similarities with that of UFs that have been reported to exhibit regions of radial and circumferential cell orientation ${ }^{33}$. Moreover, the cell-free zone of SMC clusters appears to resemble the core region of atherosclerotic plaque-like mounds in cultures of aortic SMCs reported back in $1975^{18}$ as well as the loose, water-laden myxoid tissue alternately called "necrotic core" in UFs ${ }^{34}$. It would be particularly interesting to probe if SMC clusters may serve as a useful in vitro model for studying UF development and density-driven SMC hypertrophy and hyperplasia.

\section{Materials and Methods}

\section{Cell culture}

Bovine aortic smooth muscle cells (SMCs; Cell Applications, Inc., San Diego, CA, USA) in passages 3-10 were cultured using standard procedures in bovine SMC growth medium (Cell 
Applications) and maintained at $37^{\circ} \mathrm{C}$ and $95 \%$ air $/ 5 \% \mathrm{CO}_{2}$.

\section{SMC cluster formation and visualization}

Cells were seeded on either tissue culture-treated well plates (Costar, Corning Inc., New York, NY, USA) or fibronectin-coated glass surfaces at either "low", "medium", or "high" density, corresponding respectively to $40,000,80,000$, or 160,000 cells $/ \mathrm{cm}^{2}$. The low density is considered the baseline seeding density and yielded a confluent SMC monolayer after $\sim 24 \mathrm{~h}$. The medium density corresponds to a confluent monolayer upon cell adhesion, whereas the high density results immediately in 2 to 3 overlapping cell layers. Except for the different initial densities, all experiments were performed under the same conditions. Cellular evolution was monitored over 14 days.

Bright-field images were acquired using an inverted microscope (Nikon ECLIPSE Ti2, Tokyo, Japan) equipped with a 10X objective. The acquisition rate was 1 frame every 20 min for most observations and 1 frame every $5 \mathrm{~min}$ for the "hole development" experiments whose dynamics were faster. Images (2048 x 2044 pixels) were exported in TIFF format and analyzed using FIJI.

\section{Preparation of coated glass substrates}

Small $(7 \mathrm{~mm}$-diameter) holes were drilled into a $1 \mathrm{~cm}$-thick polydimethylsiloxane (PDMS; Sylgard-to-catalyst ratio of 10:1) sheet to accommodate thin glass coverslips. The PDMS and coverslips were sterilized in ethanol for $5 \mathrm{~min}$, dried at room temperature, plasma treated for $45 \mathrm{sec}$, and then assembled together by pressure application. The assembly was then sterilized in ethanol for $5 \mathrm{~min}$ and dried at room temperature. The $7 \mathrm{~mm}$ wells so created were coated with a $0.1 \mathrm{mg} \cdot \mathrm{mL}^{-1}$ solution of GIBCO ${ }^{\mathrm{TM}}$ Fibronectin Bovine Protein (Thermo Fisher Scientific, Boston, MA, USA) in PBS ( $\mathrm{pH}$ 7.4) for $45 \mathrm{~min}$ at room temperature. The coated surfaces were then rinsed with PBS (pH 7.4) and used within $2 \mathrm{~h}$.

\section{Preparation of coated gel substrates}

Soft gel substrates were prepared using the DOWSIL ${ }^{\mathrm{TM}}$ CY 52-276 A\&B gel formulation (Dow Corning, ratio 1:1) according to the manufacturer's specifications, leading to a gel with an elastic modulus of $1-3 \mathrm{kPa}$. The gel was poured into 48 -well plates and maintained at $75^{\circ} \mathrm{C}$ for $10 \mathrm{~h}$. The gel surfaces were sterilized in ethanol for $5 \mathrm{~min}$ and dried at room temperature before coating with a $0.1 \mathrm{mg} \cdot \mathrm{mL}^{-1}$ solution of GIBCO ${ }^{\mathrm{TM}}$ Fibronectin Bovine Protein (Thermo Fisher Scientific) in PBS ( $\mathrm{pH} \mathrm{7.4)} \mathrm{for} 45 \mathrm{~min}$ at room temperature. The coated surfaces were then rinsed with PBS (pH 7.4) and used within $2 \mathrm{~h}$.

\section{SMC cluster internal structure imaging}

The 3D organization of SMC clusters was imaged using a confocal microscope (Leica SP8, Wetzlar, Germany) equipped with a $63 \mathrm{X}$ objective. The cells were stained with DAPI and phalloidin to visualize nuclei and F-actin. Images were exported in TIFF format and analyzed using FIJI.

\section{Image analysis}

Cluster outlines were defined by their bright contours in phase contrast images. Cluster contours 
were tracked using the Analyze Particles FIJI plug-in. Cluster morphometric parameters including area, perimeter, centroid position, major axis length, and minor axis length were quantified using a custom-made MATLAB code.

\section{PIV analysis}

Particle image velocimetry (PIV) was used to obtain the velocity fields of SMC tissue. The PIV analysis was performed using the Matpiv package in MATLAB (https://www.mn.uio.no/math/english/people/aca/jks/matpiv/). A three-pass computation using a final window of $32 \times 32$ pixels $(20.8 \times 20.8 \mu \mathrm{m})$ was used. The overlap was set at 0.5 . Aberrant vectors were detected and removed from the analysis when their magnitude exceeded the local mean value by three times the standard deviation ( $\sim 5 \%$ of the data). The time interval between consecutive images was $5 \mathrm{~min}$ in the "hole development" experiments and $20 \mathrm{~min}$ in the other cases. For each frame, we computed the root-mean squared-velocity as $V_{r m s}=\sqrt{\left\langle V^{2}\right\rangle}$, where $V$ is the magnitude of the velocity and the brackets denote an average over the entire field.

\section{Immunostaining}

Samples are fixed with 4\% paraformaldehyde for $15 \mathrm{~min}$ and rinsed with PBS three times and then permeabilized with $0.1 \%$ triton solution in PBS (PBST) for $15 \mathrm{~min}$. Non-specific binding was blocked using 3\% BSA in PBST. Nuclei were stained with DAPI (SigmaAldrich, St. Louis, MO, USA; 1:20,000) and F-actin with Phalloidin594 (LifeTechnologies, Carlsbad, CA, USA; 1:200). The incubation time was $1 \mathrm{~h}$.

\section{Statistical analysis}

A minimum of three experiments were performed. All data are expressed as mean $\pm \operatorname{SEM}$. Differences were assumed to be statistically significant for $p<0.05$. Statistical comparison for the data on substrate rigidity data was performed using the Student t-test. All statistical analyses were conducted using MATLAB.

\section{Acknowledgments}

The authors acknowledge all members of the Barakat group for their constructive input to the preparation of the manuscript. This work was funded by an endowment in Cardiovascular Bioengineering from the AXA Research Fund (to A.I.B.) and a doctoral fellowship from Ecole Polyechnique (to X.W.).

\section{Conflict of interest}

The authors declare no competing or financial interests.

\section{Author contributions}

X.W. and A.I.B. conceived the study. X.W. conducted the experiments. D.G.R. developed the theoretical models. T.V. conducted the PIV analysis. X.W. analyzed the experimental results with the help of D.G.R. X.W. developed the analytical tool. X.W wrote the manuscript. All authors participated in the reviewing and editing of the manuscript. 


\section{References}

1 Marieb, E. N. \& Hoehn, K. Human anatomy \& physiology. (Pearson education, 2007).

2 Biga, L. M. et al. Anatomy \& physiology. (OpenStax \& Oregon State University, 2020).

3 Doran, A. C., Meller, N. \& McNamara, C. A. Role of smooth muscle cells in the initiation and early progression of atherosclerosis. Arteriosclerosis, thrombosis, and vascular biology 28, 812-819, doi:10.1161/ATVBAHA.107.159327 (2008).

4 Geisterfer, A., Peach, M. J. \& Owens, G. K. Angiotensin II induces hypertrophy, not hyperplasia, of cultured rat aortic smooth muscle cells. Circulation research 62 , 749-756 (1988).

$5 \quad$ Hirst, S. Airway smooth muscle cell culture: application to studies of airway wall remodelling and phenotype plasticity in asthma. European Respiratory Journa/9, 808-820 (1996).

6 Shynlova, O. et al. Myometrial Apoptosis: Activation of the Caspase Cascade in the Pregnant Rat Myometrium at Midgestation1. Biology of Reproduction 74, 839-849, doi:10.1095/biolreprod.105.048124 \%] Biology of Reproduction (2006).

7 Sanders, K. M., Koh, S. D., Ro, S., Ward, S. M. \& hepatology. Regulation of gastrointestinal motility-insights from smooth muscle biology. Nature reviews Gastroenterology and hepatology 9, 633 (2012).

8 Owen, C. \& Armstrong, A. Y. Clinical management of leiomyoma. Obstetrics and Gynecology Clinics 42, 67-85 (2015).

9 Brown, B. G., Mahley, R. \& Assmann, G. Swine aortic smooth muscle in tissue culture. Some effects of purified swine lipoproteins on cell growth and morphology. Circulation research 39, 415-424, doi:10.1161/01.res.39.3.415 (1976).

10 Ross, R., Glomset, J., Kariya, B. \& Harker, L. A platelet-dependent serum factor that stimulates the proliferation of arterial smooth muscle cells in vitro. Proceedings of the National Academy of Sciences 71, 1207-1210 (1974).

11 Albers, J. J. \& Bierman, E. L. The effect of hypoxia on uptake and degradation of low density lipoproteins by cultured human arterial smooth muscle cells. Biochimica et Biophysica Acta (BBA)-Lipids and Lipid Metabolism 424, 422-429 (1976).

12 Benzakour, O. et al. Evidence for cultured human vascular smooth muscle cell heterogeneity: isolation of clonal cells and study of their growth characteristics. Thrombosis and haemostasis 75, 854-858 (1996).

$13 \mathrm{Hao}, \mathrm{H}$. et al. Heterogeneity of smooth muscle cell populations cultured from pig coronary artery. Arteriosclerosis, thrombosis, and vascular biology 22, 1093-1099 (2002).

14 Majack, R. A. Beta-type transforming growth factor specifies organizational behavior in vascular smooth muscle cell cultures. The Journal of cell biology 105 , 465-471 (1987).

15 Walthers, C. M., Lee, M., Wu, B. M. \& Dunn, J. C. Smooth muscle strips for intestinal tissue engineering. PloS one 9, e114850, doi:10.1371/journal.pone.0114850 
(2014).

16 Endlich, N., Endlich, K., Taesch, N. \& Helwig, J. J. Culture of vascular smooth muscle cells from small arteries of the rat kidney. Kidney international 57, 2468-2475, doi:10.1046/j.1523-1755.2000.00105.x (2000).

17 Frid, M., Aldashev, A., Dempsey, E. \& Stenmark, K. Smooth muscle cells isolated from discrete compartments of the mature vascular media exhibit unique phenotypes and distinct growth capabilities. Circulation research 81, 940-952 (1997).

18 May, J. F., Paule, W. J., Rounds, D. E., Blankenhorn, D. H. \& Zemplenyi, T. The induction of atherosclerotic plaque-like mounds in cultures of aortic smooth muscle cells. Virchows Archiv B 18, 205 (1975).

19 Kobayashi, Y. et al. Effects of a GnRH analogue on human smooth muscle cells cultured from normal myometrial and from uterine leiomyomal tissues. Molecular human reproduction 3, 91-99, doi:10.1093/molehr/3.2.91 (1997).

20 Gonzalez-Rodriguez, D., Guevorkian, K., Douezan, S. \& Brochard-Wyart, F. Soft matter models of developing tissues and tumors. Science 338, 910-917, doi:10.1126/science.1226418 (2012).

21 Redon, C., Brochard-Wyart, F. \& Rondelez, F. Dynamics of dewetting. Physical review letters 66, 715 (1991).

22 Tabuteau, H., Mora, S., Ciccotti, M., Hui, C.-Y. \& Ligoure, C. Propagation of a brittle fracture in a viscoelastic fluid. Soft Matter 7, 9474-9483 (2011).

23 Gonzalez-Rodriguez, D. et al. Detachment and fracture of cellular aggregates. Soft Matter 9, 2282-2290 (2013).

24 VanDijk, A. M., Wieringa, P. A., van der Meer, M. \& Laird, J. D. Mechanics of resting isolated single vascular smooth muscle cells from bovine coronary artery. American Journal of Physiology-Cell Physiology 246, C277-287, doi:10.1152/ajpcell.1984.246.3.C277 (1984).

25 Douezan, S. et al. Spreading dynamics and wetting transition of cellular aggregates. Proceedings of the National Academy of Sciences 108, 7315-7320, doi:10.1073/pnas.1018057108 (2011).

26 Cochet-Escartin, O., Ranft, J., Silberzan, P. \& Marcq, P. Border forces and friction control epithelial closure dynamics. Biophysical journal 106, 65-73 (2014).

27 Yang, J., Clark Jr, J. W., Bryan, R. M., Robertson, C. \& physics. The myogenic response in isolated rat cerebrovascular arteries: smooth muscle cell model. Medical engineering 25, 691-709 (2003).

28 Douezan, S., Dumond, J. \& Brochard-Wyart, F. Wetting transitions of cellular aggregates induced by substrate rigidity. Soft Matter 8, 4578-4583 (2012).

29 Douezan, S. \& Brochard-Wyart, F. Active diffusion-limited aggregation of cells. Soft Matter 8, 784-788, doi:10.1039/C1SM06399E (2012).

30 Duclos, G., Garcia, S., Yevick, H. \& Silberzan, P. Perfect nematic order in confined monolayers of spindle-shaped cells. Soft matter 10, 2346-2353 (2014).

31 Elsdale, T. R. Parallel orientation of fibroblasts in vitro. Experimental cell research 51, 439-450, doi:10.1016/0014-4827(68)90134-1 (1968).

32 Kemkemer, R., Kling, D., Kaufmann, D. \& Gruler, H. J. T. E. P. J. E. Elastic properties 
of nematoid arrangements formed by amoeboid cells. The European Physical Journal E 1, 215-225 (2000).

33 Flake, G. P. et al. The natural history of uterine leiomyomas: light and electron microscopic studies of fibroid phases, interstitial ischemia, inanosis, and reclamation. Obstetrics and gynecology internationa/ 2013 (2013).

34 Murase, E., Siegelman, E. S., Outwater, E. K., Perez-Jaffe, L. A. \& Tureck, R. W. Uterine Leiomyomas: Histopathologic Features, MR Imaging Findings, Differential Diagnosis, and Treatment. Radiographics 19, 1179-1197, doi:10.1148/radiographics.19.5.g99se131179 (1999). 